\title{
Estudio de la exitosa gestión de una institución de formación profesional peruana desde el enfoque de sistemas de Stafford Beer
}

\section{Study of successful management of a training institution Peruvian from system approach of Stafford Beer}

\author{
Julio Ernesto Quispe Rojas ${ }^{1}$, Julio Enrique Quispe Tuesta ${ }^{2}$ \\ ${ }^{1}$ Departamento Académico de Computación y Electrónica de la Facultad de Ciencias Físicas y Matemáticas, \\ de la Universidad Nacional Pedro Ruiz Gallo, Av. Juan XXIII No. 391 - Lambayeque - Perú. \\ ${ }^{2}$ Celeritech Solutions SAC SAP Partner, Av. Víctor A. Belaunde No. 181- Oficina 704 San Isidro, Lima - Perú.
}

\section{RESUMEN}

El modelo de sistema viable - MSV, propuesto por Stafford Beer, permite el estudio de las organizaciones desde un enfoque que privilegia la autonomía, el autocontrol, el flujo de la información, el monitoreo, la normalización, entre otros aspectos importantes para la gestión de una organización, permitiendo identificar si el sistema es viable.

Con el modelo MSV de Stafford Beer se realizó estudios a empresas bajo este enfoque en los años setenta y ochenta del siglo pasado, como el Proyecto Cybersyn en Chile, y actualmente con el desarrollo tecnológico de las TICS y la Automatización Industrial, ha sido relanzado por investigadores del enfoque de sistemas y de la cibernética organizacional, reiniciando actividades con este modelo en proyectos como el proyecto experimental para el sistema nacional ruso de innovación (NIS) del Instituto de Física y Tecnología de Moscú a partir del 2004.

El estudio se realiza a una prestigiosa Institución de Formación Profesional Peruana, de cobertura nacional, con catorce sedes zonales y más de cuarenta centros de formación profesional en todo el Perú, dedicada a la formación profesional técnica, a nivel de operarios u operadores y técnicos de mando medio, actividades de capacitación continua y capacitación en las empresas, para el sector industrial, e incluye la identificación de los componentes, sus principales relaciones y los exitosos resultados que ostenta la gestión de esta organización.

Los resultados más sobresalientes de esta Institución son: Es la primera en su género que ha logrado obtener certificaciones ISO 9001, para la gestión de la calidad, ISO 14001, para la gestión ambiental, y OHSAS18001, para la gestión de la seguridad y salud ocupacional; La inserción laboral de los egresados es muy alta, el $69 \%$ de esta trabajando en la especialidad que estudio y el $9 \%$ en una ocupación afín a su especialidad, y está considerada dentro de los 20 programas más exitosos de capacitación laboral en el mundo, de acuerdo al estudio realizado por la Agencia de Estados Unidos para el Desarrollo Internacional USAID.

La aproximación del Modelo MSV en el SENATI, muestra su utilidad para identificar los componentes y sus relaciones entre ellos y el entorno, y determinar si el sistema es viable, que se puede interpolar a partir de los resultados, que en el caso del SENATI, demuestra una gestión exitosa

El modelo favorece el estudio de problemas complejos como la gestión de organizaciones de gran envergadura, en que la autonomía, la calidad de la Toma de decisiones, el monitoreo y la normalización pueden ser decisivos para la sobrevivencia, el crecimiento y el desarrollo, y así, aplicando el enfoque los sistemas viables, basados en los principios de la Cibernética, se puede tener una gestión exitosa, llegando a ser una institución de Excelencia, aun cuando la aplicación de dichos principios no haya sido previamente identificado como tal, o quizás, se conocía con otro nombre, que puede ser el caso de SENATI.

Descriptores: cibernética, complejidad, entorno, modelo de sistema viable, formación profesional. 
ECIPERÚ

\section{ABSTRACT}

The viable system model - VSM proposed by Stafford Beer, allows the study of organizations from an approach that favours' autonomy, self-control, information flow, monitoring, standardization, among other important aspects of management an organization, allowing you to identify if the system is viable.

The study was conducted at a prestigious training institution Peruvian national coverage, with fourteen area offices and over forty professional training centres' throughout Peru, dedicated to technical education at the level of operator or operators and technicians middle management, training and continuous training in companies for the industrial sector, including the identification of components, their key relationships and successful results that shows the management of this organization.

The main results of this institution are: It is the first of its kind that has achieved ISO 9001 certifications for quality management, ISO 14001, environmental management and OHSAS 18001 for safety management and occupational health ; The employability of graduates is very high, $69 \%$ of this working in the specialty study and $9 \%$ in an occupation related to their specialty, and is considered among the 20 most successful programs of job training in the world, according to study by the U.S. Agency for International Development - USAID.

With the MSV model of Stafford Beer companies are studied under this approach in the seventies and eighties of last century, as Cybersyn Project in Chile, and now with the technological development of ICTs and Industrial Automation, has been relaunched by scholars of the systems approach and organizational cybernetics, restarting activities researchers identified this model as the pilot project for the Russian national innovation system (NIS) of the Institute of Physics and Technology in Moscow since 2004.

The MSV model approach in SENATI, shows its usefulness for identifying components and their relationships between themselves and the environment, and determine if the system is viable, which can interpolate from the results, which in the case of SENATI are demonstrates the successful management

The model promotes the study of complex problems such as managing large organizations, in which autonomy, quality of decision making, monitoring and standardization may be decisive for the survival, growth and development, and so applying the systems approach viable, based on the principles of cybernetics, you can have a successful administration, becoming an institution of excellence, even if the application of these principles has not been previously identified as such, or perhaps, known by another name, which may be the case SENATI.

Keywords: cybernetics, complexity, environment, viable system model, training.

\section{INTRODUCCIÓN}

El problema que tienen muchas organizaciones de cobertura nacional es que no se relacionan con los entornos cercanos de sus sedes descentralizadas, y por ello no conocen las necesidades y los problemas de las empresas y de los miembros la comunidad, y no aprovechan las oportunidades de satisfacer los requerimientos de los clientes activos y potenciales, y dejan de obtener los beneficios tanto económicos como de prestigio por los servicios que podrían brindar.

Las sedes descentralizadas, de esas organizaciones no tienen los niveles autonomía suficiente para la gestión de las actividades requeridas por su entorno, las organizaciones no cuentan con los sistemas de la normalización y el monitoreo de la gestión, entre otros aspectos.

Para comprender como se pierde de vista los requerimientos del entorno y se desaprovecha la capacidad de la organización, analicemos el siguiente caso.

Veamos que ocurre en unas partidas simultáneas de ajedrez, donde un maestro internacional del deporte ciencia se enfrenta a diez jugadores de la liga de primera; para que vigilen las piezas y las ubiquen en el lugar donde el maestro internacional 
les indique, le ayudan diez jugadores de la liga de primera. Como es de suponer, existe el reloj de control de tiempo, es muy probable que el maestro internacional pueda controlar el juego y ganar todas las partidas sin llegar al límite del tiempo, debido a que las combinaciones o jugadas de cada uno de los adversarios ha sido respondida con un nivel superior por el maestro internacional, la variedad generada por los adversarios ha sido absorbida por la variedad generada por el maestro.

Consecuentemente, la variedad es una medida de las combinaciones que en cada jugada puede realizar un jugador, luego, la variedad es la medida de la complejidad del juego; cuanto más capaz y experimentado es el jugador, mayor es la variedad que podrá generar, y por lo tanto, para derrotarlo se requiere generar una variedad mayor aun.

Si el maestro internacional quisiera jugar unas partidas simultaneas con seis maestros nacionales y cuenta con la ayuda de otros seis maestros nacionales encargados de vigilar las piezas y realizar los movimientos que el maestro internacional les indique, lo que podría ocurrir es que pierda algunas partidas (digamos dos) por cumplimiento de tiempo, pierda otras dos porque los maestros nacionales le ganan la partida y por ultimo gane las otras dos. Podría haber ocurrido que uno de los maestros nacionales que le ayudaba, se excusara de seguir ayudándolo, antes de acabar la partida, porque ve que si el jugara, según él, ya le habría ganado al adversario.

\section{¿Qué ha pasado?}

Ahora, la variedad generada por el maestro internacional no ha podido absorber toda la variedad generada por los maestros nacionales; luego la variedad total generada por los maestros nacionales fue mayor que la variedad generada por el maestro internacional.

¿Ocurre algo similar a las partidas simultáneas del maestro internacional contra maestros nacionales en la gestión de las organizaciones de cobertura nacional con dirección centralizada?

$\mathrm{Si}$, se tiene jugadores para que vigilen y realicen las jugadas indicadas por el maestro internacional, que es la dirección centralizada de la organización, administrando el presente y el ambiente interno sin dar paso a la capacidad, a la creatividad y a la experiencia de los jugadores para enfrentarse al futuro y el ambiente externo con autonomía y los recursos necesarios para una buena gestión.
La solución a esta problemática se tiene que dar en términos de un manejo adecuado de la variedad, de tal forma que la variedad generada por la organización en su conjunto, dirección general, dirección de las sedes descentralizadas y miembros de los diferentes niveles, sea mayor que la variedad generada por el entorno, el mundo globalizado y competitivo, que no tiene fronteras.

El desafío emergente de la gestión de las organizaciones de cobertura nacional, es resolver el problema del centralismo y la asignación de recursos, el monitoreo y no limitación en las decisiones, la normalización y el soporte a la gestión, los vínculos entre los componentes de la organización, para ello se requiere de un enfoque que contemple el manejo de la variedad generada por los diferentes componentes de la organización y su entorno, e involucre las especificidades de cada organización y su entorno.

Este estudio del Modelo de Sistema Viable - MSV de Stafford Beer, se realiza a una prestigiosa Institución de Formación Profesional Peruana, muy exitosa, e incluye la identificación de los componentes, sus principales relaciones y los exitosos resultados que ostenta la gestión de esta organización, que ha aplicado los principios del modelo MSV, deliberadamente o como parte de sus esfuerzos por ser excelentes.

\section{MSV UNA RESPUESTA VIABLE}

El tratamiento de la variedad es abordado con profundidad por Stafford Beer quien desde su enfoque cibernético de la Administración, formula un modelo de diagnostico organizacional conocido como Modelo de Sistema Viable - MSV (Beer, 1977, $1979,1981,1985)$ que permite enfrentar el estudio de las organizaciones con criterios de autonomía y descentralización mediante el manejo de la variedad y la variedad requerida conocida como Ley de Ashby (Ashby, 1986): "solo la variedad puede absorber a la variedad", lo cual se confirma en el caso del juego de ajedrez antes indicado.

Norbert Wiener acuñó el nombre de Cibernética en 1947 y la definió como la ciencia de la comunicación y el control, en los animales y en las maquinas, y Stafford Beer desarrolla esta definición indicando que la Cibernética estudia la corriente de la información que rodea a un sistema y el modo en que esta información es utilizada por el sistema para controlarse así mismo (Beer, 1982).

La Cibernética tiene como objetivo principal el estudio de la interacción entre los sistemas 
gobernantes (control) y los sistemas gobernados (operaciones), mediante procesos de retroalimentación definiéndose como la ciencia de los mecanismos autorregulados. Es aplicable a los procesos homeostáticos del universo biológico y a los automatizados del autocontrol de los ordenadores, como indica Bruno Lussato (1976).

Leonid Ototsky, Jefe del Sistema de Gestión e Integración de la MIPT de Moscú, en su investigación Stafford Beer y el Sistema Viable en el siglo XXI - Moscú 2004, dice que la extraordinaria expansión de Internet ha intensificado una serie de problemas sociales, metodológicos y tecnológicos, y las actuales capacidades y desarrollos del Internet, están sobrepasando el alcance visionado por sus creadores. Decisiones en el campo de las TICs para disminuir las horas hombre, y para crear y desarrollar sistemas, está siendo investigado en todos los frentes. Un ejemplo es el desarrollo de la computación autonómica de la IBM, que facilita y automatiza muchos de los sistemas de gestión.[1]

Las potencias en ventas de ERP, como SAP y Oracle, están creando fuertes herramientas analíticas para apoyar a las empresas más viables. Algunos ejemplos son la supervisión de actividades económica de Oracle y el Gestor Estratégico de Empresas de SAP; muchas características del VSM de Beer, como por ejemplo: las alarmas en tiempo real, auto-corrección, auto-protección, autoadaptación y tableros de instrumentos gráficos, están emergiendo en estos nuevos sistemas, estos se relacionan directamente con el nuevo software "The Viable Software" de Charles Herring.

Las tecnologías actuales están haciendo posible la implementación de las ideas del VSM, que a la hora de la puesta en práctica del proyecto Cybersyn, hace 30 años en Chile, no se disponía, y hoy en día existe una nueva fuerza de interés en Rusia, con el propósito de utilizar la herencia "instrumental" de Stafford Beer; el proyecto experimental para el sistema nacional ruso de innovación (NIS) del Instituto de Física y Tecnología de Moscú, está basado en los sistemas viables de Beer y es el inicio de proyectos para gestión de la innovación en Rusia. (Ototsky, 2004)

Pérez Ríos (2008) dice que en las últimas décadas el mundo ha cambiado de forma vertiginosa, la diversidad de factores que han intervenido en el cambio y su interrelación hace que la complejidad implícita en dicho cambio sea también formidable, y podemos resumir que el estado de la cuestión afirmando que tanto las organizaciones en general, como las empresas en particular, se enfrentan a problemas de una gran complejidad dinámica.[2]

Pérez Ríos y Leonid Ototsky, son dos de los exponentes del pensamiento sistémico que han relanzado el Modelo de los Sistemas Viables de Stafford Beer, y destacado su utilidad en el contexto actual de permanente cambio.

El Modelo de Sistemas Viable-MSV, propuesto por Beer es particularmente adecuado cuando se requiere enfocar la autonomía y el control en una organización, para lograr un funcionamiento que responda a los cambios del entorno y a las necesidades operativas.

El Modelo de Beer identifica lo que se llama niveles de Recursión. Siendo el nivel $X$ un determinado nivel de Recursión, la misma estructura y relaciones que se puede encontrar entre los componentes de este nivel, se repite al interior de los componentes operativos de dicho nivel. Por tanto si en el nivel $X$, se tiene dos componentes operativos (sistemas 1), en el interior de cada componente operativo, que llamaremos nivel $\mathrm{Y}$, se repite la misma estructura y relaciones que en $X$, el nivel $Y$ es un componente de un nivel de Recursión superior $X$.

Esto se observa en la figura No.1, en el que el sistema que estamos analizando se convierte en Sistema 1 de una recursión inmediata superior.

El modelo de Stafford Beer, se muestra en la figura No. 1, tiene cinco sistemas. El Sistema 5, involucra la cultura organizacional y los valores de los directivos; el Sistema 4 relacionado con la planificación y dedicado al manejo del futuro y el entorno; el sistema 3 que se ocupa de las actividades administrativas, esto es el corto plazo y el ambiente interno; el Sistema 2 de regulaciones que comprende las normas, procedimientos $y$ estándares de gestión. Finalmente, el Sistema 1 que se dedica a las actividades operativas de la empresa. 


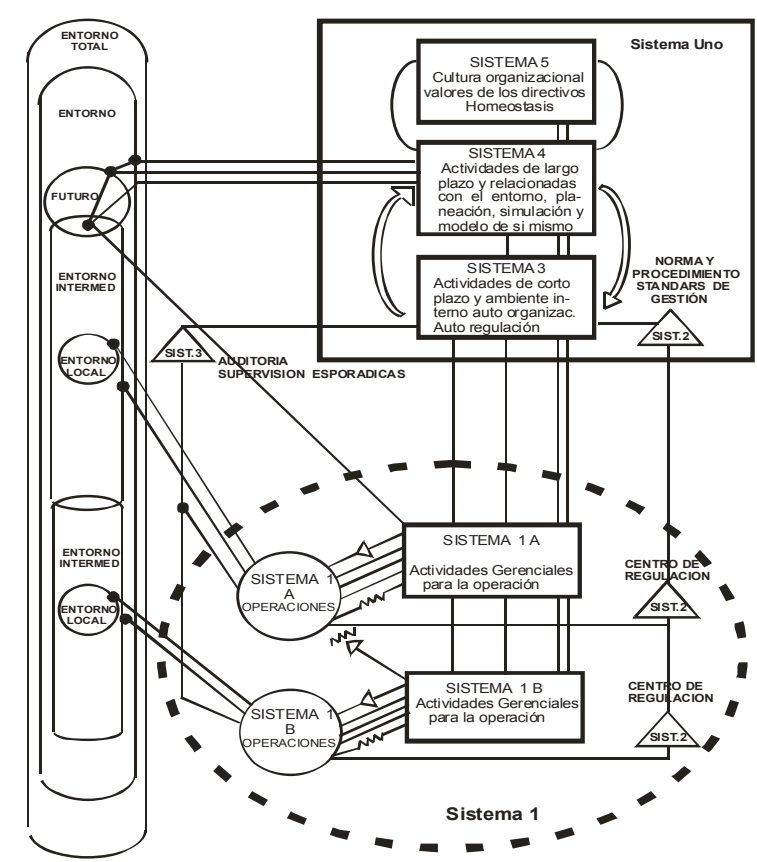

Figura 1. Modelo del Sistema Viable - MSV

El Sistema 1 es aquel que se encarga de las operaciones rutinarias de la organización y que puede sobrevivir aun cuando se desligue del Sistema General. Existe una interacción entre los componente del Sistema 1, que son: la Gerencia de operaciones que da las directivas al área de operaciones, el área de operaciones encargada de las actividades operativas; y el entorno; que es el ambiente externo en que se desenvuelve este sistema, tal como se muestra en la Figura No. 2.

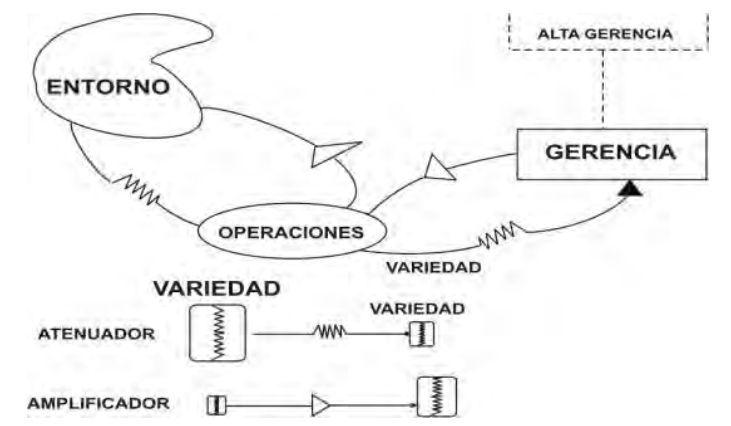

Figura 2. Relaciones atenuador amplificador

GERENCIA: Todas las actividades gerenciales del área operativa.

OPERACIONES: Todas las actividades operativas que realiza el sistema.

ENTORNO: Ambiente con el que interaccionan las actividades operativas.
El Modelo de Beer propone compensadores de variedad, como el atenuador que reduce la variedad, esto es, convierte la alta variedad de un Terminal de entrada en baja variedad en un Terminal de salida; también considera el amplificador que cumpla la función inversa. El análisis del lazo homeostático (la homeóstasis es la característica de los organismos vivos por lo cual se mantienen constantes, dentro de los limites fisiológicos, determinadas variables criticas) es uno de los fundamentos del Modelo de Beer y consiste en lograr el equilibrio dinámico entre los dos bloques; uno de alta variedad y el otro de baja variedad, compensando las diferencias de variedad con el atenuador y el amplificador, para llegar al equilibrio.

Para que un Sistema 1 sea un Sistema Viable, es necesario que exista un balance de variedad o equilibrio fundamental de variedad entre la variedad generada por los componentes del Sistema1: manejo del entorno, operaciones y gerencia de

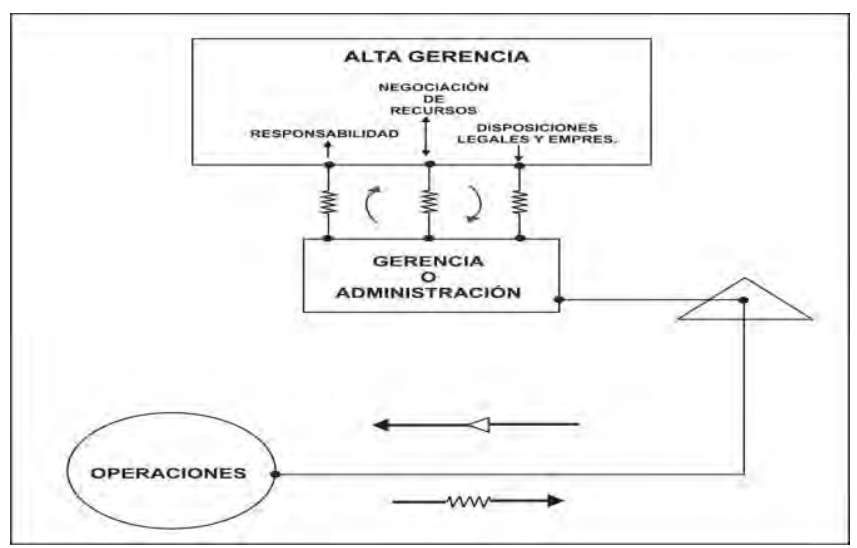

operaciones y la variedad generada por la alta gerencia, principalmente a través de los vínculos fundamentales, ya que la interacción de los entornos no depende de la alta gerencia.

Figura 3. Vínculos fundamentales

Y los vínculos fundamentales cuando no son adecuadamente dimensionados llevan a una asfixia del Sistema 1 por encontrarse entre las presiones del entorno y las restricciones de la empresa. Para solucionar ello se debe realizar el dimensionamiento de los vínculos fundamentales con la participación de los miembros del Sistema 1, tal como se muestra en la Figura 3, permitiendo de esa forma otorgar el grado de autonomía que requiere el Sistema 1 para una exitosa gestión. 


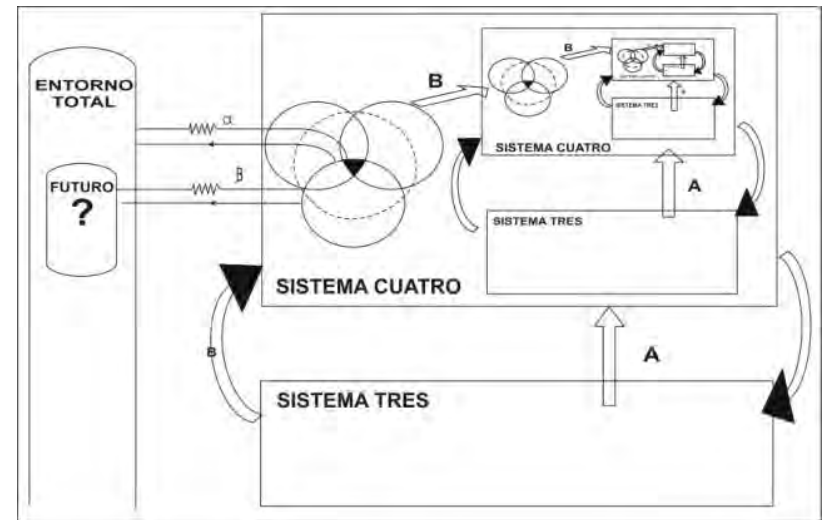

Figura 4. Simulación y estrategia en el sistema 4

El Modelo de Beer considera también, a través del Sistema 4, la planeación, el futuro y el entorno como un componente importante que le permite tener una referencia de sí mismo y analizar mediante la simulación los efectos de las estrategias y decisiones; y así mismo, evaluarlas y realizar, mediante la retroalimentación, las variantes necesarias para responder a los cambios del entorno y del ambiente interno, como en la Figura 4.

\section{EI SENATI UN SISTEMA VIABLE}

El Servicio Nacional de Adiestramiento en Trabajo Industrial - SENATI, es una organización de cobertura nacional con una sede central en la Capital del Perú, Lima, y catorce sedes de Direcciones Zonales, cuarenta y cinco sedes descentralizadas en toda la República del Perú, y en esta conformado, siguiendo a Beer, por cinco sistemas: El Sistema de Valores, el Sistema de Planeamientos, el Sistema Administrativo, el Sistema de Normas, Procedimientos y Estándares de Gestión y el Sistema Operativo[3]

El Sistema 5 está formado por las Políticas del Consejo Nacional del SENATI, que incluye la Axiología, los Principios y Fines del SENATI, y los valores de los directivos, los Planes de Desarrollo del País.

EI Sistema 4 del SENATI, lo conforma el Consejo Nacional, la Dirección Nacional, la Secretaria del Consejo Nacional, el Representante de la Dirección para el Sistema de Gestión Integrada - SIG y el Comité Nacional de Gestión.

El Sistema 3, lo conforman las siete gerencias centrales del SENATI: Gerencia de Recursos Humanos, Gerencia Académica, Gerencia Técnica, Gerencia de Finanzas, Gerencia de Logística y
Administración, Gerencia de Desarrollo y Gerencia Legal.

El Sistema 2, está formado Auditoría Interna y las Normas del Sistema de Gestión Integrada SIG, formado por Gestión de Calidad, Gestión Ambiental y Gestión de Seguridad y Salud Ocupacional

El Sistema 1, Lo forman las catorce Zonales del SENATI, cuyas sedes están en las principales ciudades del Perú. La Zonal Lima - Callao, es la sede que atiende a la capital del Perú y la Provincia Constitucional del Callao, las otras trece zonales son: Piura - Tumbes, Lambayeque - Cajamarca Norte, La Libertad, Cajamarca Sur - Amazonas, San Martín, Loreto, Ancash, Ucayali - Huánuco, Junín Pasco - Huancavelica, Ica - Ayacucho, Cusco Apurímac - Madre de Dios, Arequipa - Puno, y Moquegua - Tacna.

La Organización Nacional del Servicio Nacional de Adiestramiento en Trabajo Industrial - SENATI, la podemos definir como el nivel de Recursión $X$, y cada Zonal como un nivel de Recursión Y, y la Zonal tiene una estructura similar a la Organización Nacional.

Una Zonal tiene un Sistema 5 formado por la Política Nacional, la cultura organizacional de la sede, los valores de los directivos zonales; un Sistema 4 formado por el Consejo Zonal, la Dirección Zonal, el Comité de Gestión Zonal y las Comisiones Consultivas de Empleadores; un Sistema 3, formado por la Dirección Zonal y Oficina de Administración; Un Sistema 2, formado por las normas del Sistema de Gestión Integrado - SIG y el Sistema Informático de Gestión - SINFO; y uno o varios Sistemas 1, que están formados por los Centros de Formación Profesional y los Proyectos Especiales de Informática y idiomas, llamados Programa Nacional de Informática - PNI y Centro de Idiomas.

Una Zonal puede tener uno o más Centros de Formación Profesional, y se puede considerar como un nivel de Recursión Z, y está formado por el Jefe del Centro de Formación Profesional, el Comité de Gestión y el Equipo de Formadores o Instructores que están encargados de la formación profesional de los estudiantes, del mantenimiento del equipamiento para la formación profesional y de la gestión de los recursos para la formación profesional. 
EI SENATI tiene una Sede Central, donde está El Consejo Nacional y La Dirección Zonal; en el mismo local tiene su sede la Zonal Lima - Callao, donde está la Dirección Zonal Lima - Callao y el Centro de Formación Profesional Principal del SENATI, que es parte de la Zonal Lima Callao, que además tiene otros 10 Centros de Formación Profesional: Lince, San Juan de Lurigancho, Villa El Salvador, San Martín de Porres, Surquillo, San Juan de Miraflores, Callao - Ventanilla, Lima Cercado, Cañete y Huacho.

En la sede Central, ubicada en el Distrito de Independencia de Lima, la Capital del Perú, dependiendo de la Dirección Zonal de Lima Callao, también se tiene como parte operativa, nivel de Recursión Z, el Centro PYME, el Programa Nacional de Informática - PNI, el Centro de Idiomas,

EI SENATI, tiene cuarenta y cinco Centros de Formación Profesional a Nivel Nacional, algunas Zonales tienen un solo Centro de formación Profesional y otras más de una; algunas se encuentran en la misma sede de la Dirección Zonal y otras en sedes ubicadas en otras localidades.

\section{LAS MUESTRAS DE LA REALIDAD}

EI SENATI- Servicio Nacional de Adiestramiento en Trabajo Industrial es una institución que tiene por finalidad proporcionar formación profesional y capacitación para la actividad industrial manufacturera y para las labores de instalación, reparación y mantenimiento realizadas en las demás actividades económicas

Motivados por el hecho que la formación profesional y la educación técnica tradicionales no otorgaban las calificaciones requeridas por la actividad productiva moderna, los empresarios de la Sociedad Nacional de Industrias decidieron en 1960, promover la creación de una institución destinada específicamente a desarrollar las aptitudes humanas para el desempeño competente de las ocupaciones profesionales de la actividad industrial manufacturera y de las labores de instalación, reparación y mantenimiento; para cuyo financiamiento se impusieron un auto gravamen 0 contribución económica mensual.

En atención a esta iniciativa de los empresarios, el SENATI fue creado el 19 de Diciembre de 1961 mediante la Ley $N^{\circ} 13771$.
La metodología de enseñanza, más caracterizada del SENATI es el "aprender haciendo " y en condiciones reales de producción. La formación en ocupaciones técnicas operativas se realiza mayormente con el Sistema Dual SENATI-Empresa, que se caracteriza por alternar actividades en los Centros de Formación Profesional con aprendizaje práctico dentro de las empresas, son cerca de 8600 las empresas que, a nivel nacional, participan en el Aprendizaje Dual conducido por el SENATI.

En función de los diferentes requerimientos que se presentan en la estructura ocupacional de la actividad productiva, el SENATI ha establecido programas de formación y capacitación profesional para los siguientes niveles ocupacionales: Nivel Técnico Operativo, Nivel Técnico Medio y Nivel Técnico Superior

Desde el año 2000, el SENATI trabaja en el fortalecimiento y consolidación de su Sistema Integrado de Gestión, con acciones orientadas tanto a la gestión de la Calidad, Ambiental y a la Seguridad y Salud Ocupacional. En Latinoamérica, el SENATI es la primera institución en su género, que ha logado obtener las certificaciones: ISO 9001, para la Gestión de la Calidad, ISO 14001, para la Gestión Ambiental y OHSAS 18001, para la Gestión de la Seguridad y Salud Ocupacional. Dichas certificaciones, tienen un alcance a nivel nacional, en todos sus Centros de Formación Profesional. De esta forma el SENATI se mantiene a la vanguardia, al aplicar normas reconocidas internacionalmente en la gestión de sus actividades y servicios que ofrece a nivel nacional y al actualizar los contenidos curriculares de sus programas de Formación y Capacitación Profesional en base a los estándares.

La función principal del SENATI es impartir formación y capacitación profesional para la actividad industrial manufacturera y para las labores de instalación, reparación y mantenimiento. Asimismo, desarrolla servicios técnicos, para el cumplimiento de sus funciones, el SENATI ha establecido un Sistema de Formación y Capacitación Profesional que responde a las reales demandas de la actividad productiva. Este sistema tiene las siguientes características:

Participación de los empresarios en los órganos de dirección y en los procesos de planificación y desarrollo de la formación profesional: aproximadamente 250 empresarios, a nivel nacional.

Programas, perfiles profesionales, contenidos curriculares, metodologías y formas organizativas 
que siguen el enfoque de formación profesional por competencias laborales concretas.

Personal técnico-docente y de gestión con experiencia industrial, capacitado y perfeccionado en países altamente desarrollados de América, Europa y Asia.

Centros de Formación y Capacitación profesional con equipamiento moderno y permanentemente actualizado.

Tecnología educativa innovadora en materia de formación profesional técnica, con reconocimiento a nivel nacional e internacional.

Experiencia en la gestión económico-financiera acorde con las técnicas modernas de calidad, productividad y rentabilidad.

La naturaleza jurídica del SENATI, define como una persona jurídica de derecho público con autonomía técnica, pedagógica, administrativa y económica, y con patrimonio propio, se rige por las disposiciones de la Ley No. 26272 (promulgada el 1 ero de Enero del año 1994), por su Reglamento de Organización (aprobado el Decreto Supremo 05-94TR) y las normas que aprueba su Consejo Nacional y no está comprendido en los Sistemas Administrativos del Sector Público y no le son de aplicación sus normas, con excepción del control pertinente de la Contraloría General de la República.

EI SENATI financia sus actividades mediante Recursos propios que genera por los diversos servicios que brinda, incluida la Formación y Capacitación Profesional y la Asistencia Técnica, La contribución económica que aportan mensualmente las empresas de más de 20 trabajadores, respecto al personal dedicado a la actividad industrial manufacturera y a las labores de instalación, reparación y mantenimiento. El monto de la contribución es equivalente al $0.75 \%$ del total de las remuneraciones que paguen las empresas a sus trabajadores. Las empresas aportantes tienen derecho a formar gratuitamente a futuros trabajadores operativos, así como capacitar a sus trabajadores en servicio, y los provenientes de la Cooperación Técnica Internacional y nacional

EI SENATI cuenta con 14 Direcciones Zonales que agrupan 45 Centros de Formación Profesional a lo largo del todo el territorio peruano.

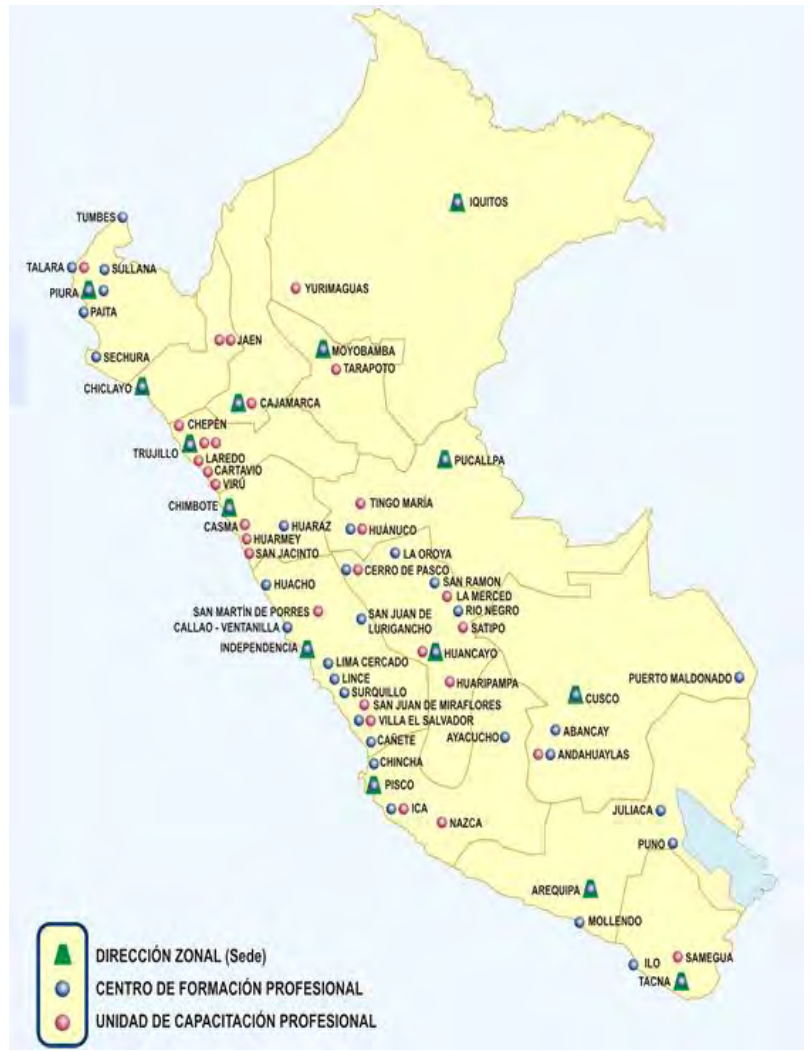

Figura 5. Ubicación de las direcciones zonales, CEF y UCP.

Los procesos del SENATI, se han identificado en la siguiente clasificación:

PROCESOS DE DIRECCIÓN (5): Definen y despliegan la política y la estrategia de la institución, constituyen el marco de referencia para los demás procesos.

PROCESOS OPERATIVOS (3): Constituyen la secuencia de valor agregado, desde la determinación de necesidades hasta el servicio posventa.

PROCESOS DE SOPORTE (7): Son los que dan apoyo, fundamentalmente a los procesos operativos

La última Certificación del Sistema Gestión Integrado del SENATI se ha realizado en el 2009 por la certificadora Bureau Veritas Certification

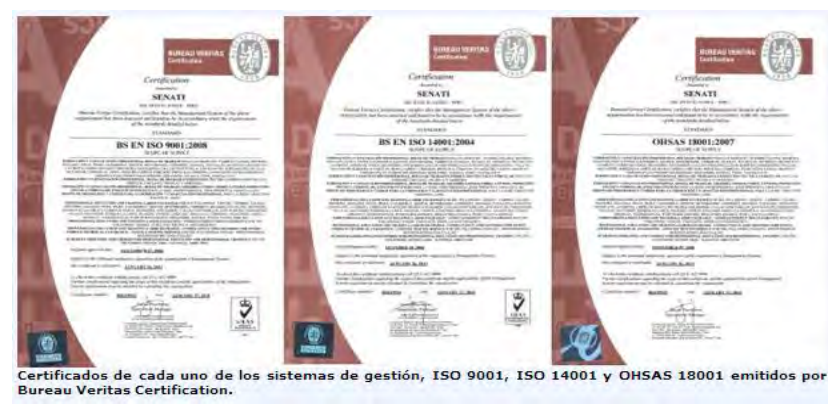


Figura 6. Certificados de Calidad, Ambiental y Seguridad y SO.

En un estudio sobre la empleabilidad de los egresados del SENATI, realizado en el 2001, por Aiga Von Hippel, de la universidad Ludwig Maximilian de Munich, Alemania, con apoyo de la GTZ de Alemania, se obtuvieron los siguientes resultados: De la muestra tomada, 90 egresados, el $69 \%$ estaba trabajando en la especialidad que ha estudiado, el $9 \%$ trabajaba en una especialidad afín y el $22 \%$ no trabajaba.

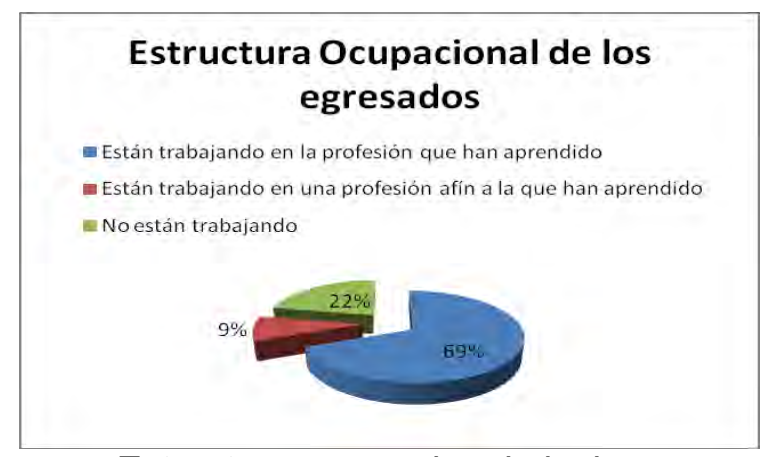

Figura 7. Estructura ocupacional de los egresados del SENATI.

La satisfacción en el puesto de trabajo, más del $62 \%$ se encontraba muy satisfecho o satisfecho, 15 $\%$ el insatisfecho y resto, no estaba contento con su trabajo.

En lo referente a la utilidad de los conocimientos adquiridos, se nota en las siguientes figuras, la gran utilidad de los conocimientos adquiridos en el SENATI y en la empresa

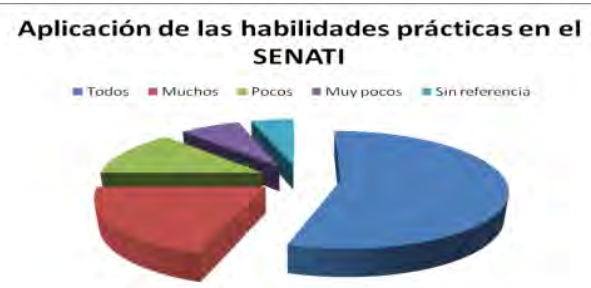

Figura 8. Uso de las habilidades prácticas adquiridas en SENATI.

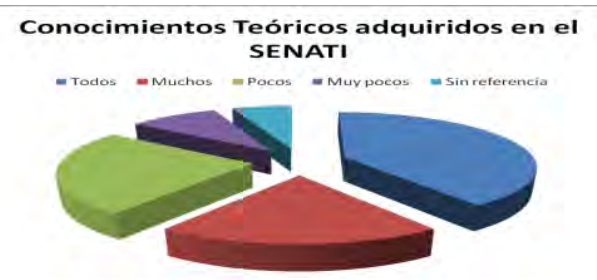

Figura 9. Uso de los conocimientos teóricos adquiridas en SENATI.

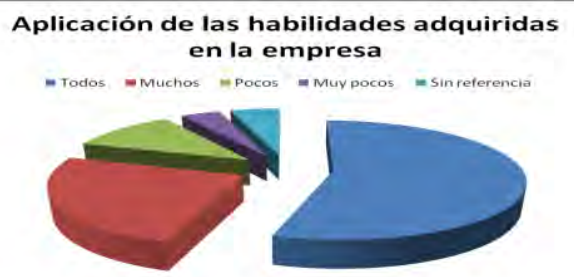

Figura 10. Uso de las habilidades prácticas adquiridas en la Empresa.

EI SENATI está considerado dentro de los veinte programas más exitosos de capacitación laboral en el mundo, de acuerdo al estudio realizado por la Agencia de Estados Unidos para el Desarrollo Internacional - USAID.

Por Ley Promulgada el 29 de Marzo de 2011, los técnicos egresados del Servicio Nacional de Adiestramiento en Trabajo Industrial - SENATI, al igual que las universidades, podrán obtener sus títulos profesionales a nombre de la Nación. Los profesionales técnicos egresados de los 45 centros que tiene el SENATI en el Perú, pueden realizar el trámite respectivo para obtener dicha acreditación.

Las especialidades que ofrece actualmente son: la Metalmecánica, Artes Gráficas, Trabajos en Madera, Electrotecnia, Mecánica Automotriz, Textil y Confecciones. También en Cerámica Industrial, Industria Alimentaria, Joyería, Orfebrería y Platería, Turismo, y Administración de Empresas.

\section{CONCLUSIONES Y RECOMENDACIONES}

El modelo MSV, se aplico a organizaciones en los años setenta y ochenta del siglo pasado, como el Proyecto Cybersyn en Chile, y actualmente con el desarrollo tecnológico de las TICS y la Automatización Industrial, ha sido relanzado el modelo, como en el proyecto experimental para el Sistema Nacional Ruso de Innovación (NIS) del Instituto de Física y Tecnología de Moscú el 2004.

La aproximación del Modelo MSV en el SENATI, muestra su utilidad para identificar los componentes $y$ sus relaciones entre ellos y el entorno, $y$ determinar si el sistema es viable, que se puede interpolar a partir de los resultados, en un estudio inicial; estos resultados en el caso del SENATI demuestran una gestión exitosa

Aplicando el enfoque los sistemas viables, basados en los principios de la Cibernética, se puede tener una gestión exitosa, llegando a ser una institución de Excelencia, aun cuando la aplicación de dichos principios no haya sido deliberada o identificada 
como tal, o quizás, se conocía con otro nombre, que puede ser el caso de SENATI, y se debe realizar un estudio a mayor profundidad, de carácter explicativo, con fines de destacar las acertadas estrategias y tácticas de gestión para replicar en organizaciones de diverso tipo que pretendan ser viables y exitosas.

\section{REFERENCIAS}

[1]LEONID OTOTSKY, Stafford Beer y el Sistema Viable en el Siglo XXI, Moscu 2004

[2]JOSE PEREZ RIOS, Diseño y diagnóstico de organizaciones viables, Madrid 2008

[3]SENATI, PAGINA WEB www. Senati.edu.pe.

e-mail: jquisper@unprg.edu.pe

julioqt@gmail.com 